\title{
Reinnervation of Long-Term Denervated Rat Muscle Freely Grafted into an Innervated Limb
}

\author{
BRUCE M. CARLSON*,† AND JOHN A. FAULKNER $\ddagger \S$ \\ Departments of *Anatomy, + Biology, and $\ddagger$ Physiology, and $\$$ Institute of Gerontology, University of Michigan, Ann Arbor, Michigan 48109
}

This experiment was designed to test the hypothesis that in the presence of regenerating nerve fibers longterm denervated skeletal muscle does not become reinnervated. This hypothesis was tested in rats by the transplantation of 22-month denervated extensor digitorum longus (EDL) muscles into the sites of EDL muscles in the contralateral, normally innervated legs. Two months after transplantation, the muscles contracted when stimulated via the motor nerve, and based on silver-acetylcholinesterase staining, all grafts possessed innervated motor end plates. Compared to values for control EDL muscles in old rats, the maximum force developed by standard free grafts in old rats was $19 \%$ and that of long-term denervated grafts was 7\%. For standard free grafts, nerve stimulation produced a maximum force that was $81 \%$ of that produced by direct stimulation, and for control EDL muscles in young and old rats, the values were 96 and $90 \%$, respectively. These results show that after long-term denervation rat muscles are capable of becoming functionally reinnervated, even though by the time of reinnervation the animals have attained an advanced age of 26 months. (c) 1988 Academic Press, Inc.

\section{INTRODUCTION}

A widely accepted premise in dealing with peripheral nerve injuries is that the longer the interval between denervation and the arrival of regenerating nerve fibers at a denervated muscle, the poorer the degree of motor recovery. In clinical practice the period between 12 and 24 months is viewed as a threshold beyond which successful functional reinnervation of denervated muscle decreases markedly. Sunderland ((18), p. 312) stated that reinnervation of human muscle up to 12 months after denervation can be highly successful, but he left open the option that the duration of denervation may be a factor in limiting the functional recovery of muscles. Bateman ((3), p. 12) concluded that after 12 to 18 months of denervation fewer end-plate connections form upon reinnervation of the muscle, and some muscle fibers don't respond to the new connections that do develop. Anderl ((1), p. 246; (2), p. 262) noted that the facial muscles are more resistant than limb muscles to the effects of denervation, but that after 1.5 to 2 years of denervation it is not efficient to attempt direct reinnervation of a muscle.

A number of possibilities exist for the poor reinnervation of long-term denervated muscle. One is that the regenerating nerve fibers don't find their way back to the muscle. A second is that fibrotic or other chronic degenerative changes at the neuromuscular junction preclude normal nerve-muscle interactions when regenerating nerve fibers reach the region of the neuromuscular junction (13). A third possibility is that after a long period of denervation an appropriately reinnervated muscle fiber is incapable of responding to the influence of the motor nerve.

In our experiments, a model of muscle transplantation was used to study reinnervation. The model consisted of denervating a limb muscle for almost 2 years and then grafting the denervated muscle into a normally innervated leg. We tested the hypothesis that in the presence of regenerating nerve fibers long-term denervated muscle does not become reinnervated. This hypothesis was tested by measuring contractile properties obtained by nerve stimulation, direct muscle stimulation, and morphological methods. The rats were aged by the end of this experiment. Therefore, determination of the effects of age on the contractile properties of control skeletal muscles was necessary to make appropriate interpretations of the results.

\section{MATERIALS AND METHODS}

The experiments were conducted upon 22 highly inbred (over 90 generations) male rats of the F-455 Wistar strain, maintained at the University of Michigan. As early as 60 generations of inbreeding, muscles could be grafted from one animal to another with no histological evidence of rejection (Carlson, unpublished data). The animals were anesthetized with ether during each operation. All experiments were conducted in accordance with the published guidelines of the American Physiological Society.

\section{Long-Term Denervated Series}

The right legs of two-month-old rats were denervated by removing a section of the sciatic nerve high in the 

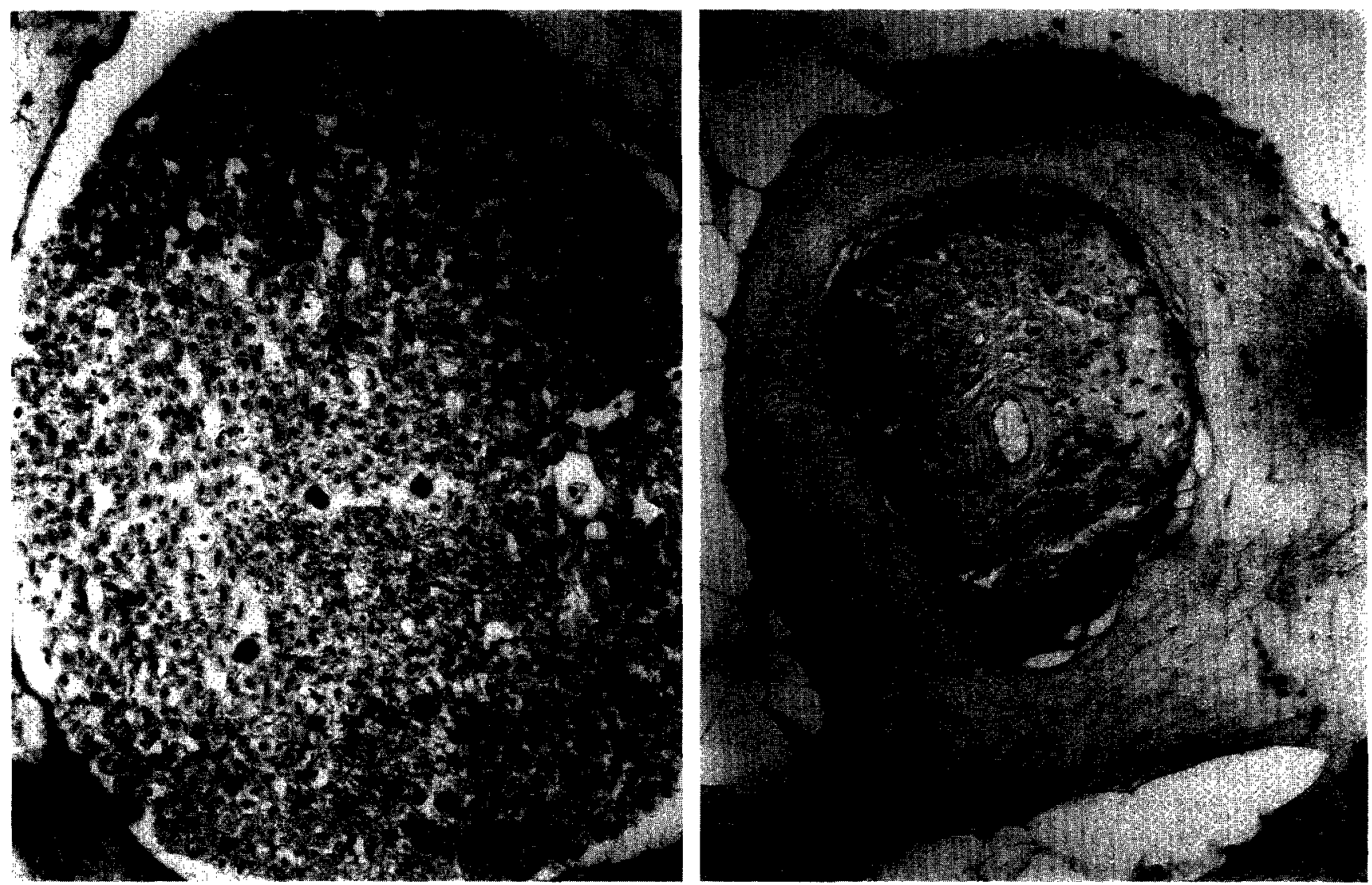

FIG. 1. Silver-stained frozen sections through the sciatic nerve of control (A) and 22-month denervated (B) legs. 181 $\times$.

thigh. In order to prevent reinnervation, a suture was tied tightly around the proximal and distal nerve stumps, and the proximal nerve stump was implanted into a nearby mass of muscle. Silver staining of segments from the distal nerve and electrical stimulation of the denervated sciatic nerve have shown that this surgical technique prevents the return of nerve fibers for an indefinite period (Fig. 1). In addition, morphological studies (electron microscopy and silver staining) of some surrounding muscles showed no evidence of reinnervation.

Five extensor digitorum longus (EDL) muscles were grafted 22 months after denervation. The right leg was opened, and the sciatic nerve was electrically stimulated. In no case did the muscles of the lower leg contract. Next, the EDL muscle in the left leg was isolated, removed, and discarded. Then the 22 -month denervated right EDL muscle (almost string-like in appearance) was removed and grafted in the site of the EDL muscle in the left leg of the same animal. The tendons of the long-term denervated EDL graft were sutured to the tendon stumps of the left EDL muscle, and the stump of the nerve to the left EDL muscle was implanted into the graft. In suturing the tendons, care was taken to ensure that remnants of muscle fibers did not remain on the tendon stumps of the host. Two months later, when the rats were 26 months old, contractile properties of the grafts were measured in situ.

\section{Histology Series}

Five long-term denervated ( 22 month) muscles were grafted in place of the EDL muscle of other rats. These grafts were removed after intervals of $5,7,10,15$, and 20 days for histological analysis of the early postoperative morphology of grafted long-term denervated muscle. Contractile properties were not measured on these grafts.

\section{Control Series}

In five normal 24-month-old rats, the right EDL muscles were removed and orthotopically grafted, with the stump of the EDL nerve implanted into the graft. Two months later the muscles in these animals were analyzed in the same manner as the long-term denervation grafts. The nonoperated left EDL muscles were also analyzed as normal controls.

Contractile properties of control EDL muscles and of short-term and long-term denervated grafts from old ( 26 month) rats were measured in situ 60 days after the grafting operation. The rats were anesthetized with so- 
TABLE 1

Structural and Contractile Properties of Control Extensor Digitorum Longus (EDL) Muscles, Standard EDL Grafts, and Grafts of Long-Term Denervated EDL Muscles of Old (26 Month) Male Wistar Rats and Control EDL Muscles from Young (4 Month) Male Wistar Rats

\begin{tabular}{lcccccr}
\hline & $L_{\mathrm{m}}(\mathrm{mm})$ & $L_{\mathrm{f}}(\mathrm{mm})$ & $M_{\mathrm{m}}(\mathrm{mg})$ & $\mathrm{X}-\mathrm{sec}\left(\mathrm{mm}^{2}\right)$ & $P_{0}(\mathrm{mN})$ & $P_{0}\left(\mathrm{~N} / \mathrm{cm}^{2}\right)$ \\
\hline $\begin{array}{l}\text { EDL muscles, adult rats } \\
(N=5)\end{array}$ & $29.6 \pm 0.9$ & $14.2 \pm 0.1$ & $166 \pm 4$ & $11.0 \pm 0.3$ & $2840 \pm 85$ & $25.8 \pm 1.2$ \\
$\begin{array}{l}\text { EDL muscles, old rats } \\
\quad(N=5)\end{array}$ & $32.2 \pm 0.66$ & $12.9 \pm 0.3$ & $157 \pm 2$ & $11.4 \pm 0.2$ & $2414 \pm 24$ & $21.2 \pm 0.9$ \\
$\begin{array}{l}\text { Standard grafts, old rats } \\
\quad(N=5)\end{array}$ & $28.8 \pm 1.2$ & $11.5 \pm 0.5$ & $74 \pm 6$ & $6.1 \pm 0.4$ & $452 \pm 68$ & $7.4 \pm 2.1$ \\
$\begin{array}{l}\text { Long-term denervated } \\
\text { grafts, old rats } \\
(N=4)\end{array}$ & $29.9 \pm 0.5$ & $12.1 \pm 0.2$ & $49 \pm 8$ & $3.7 \pm 0.5^{*}$ & $169 \pm 70^{*}$ & $4.5 \pm 2.0$ \\
\hline
\end{tabular}

Note. $L_{\mathrm{m}}=$ muscle length, $L_{\mathrm{f}}=$ fiber length, $M_{\mathrm{m}}=$ muscle mass, $\mathrm{X}$-sec $=$ total fiber cross-sectional area, $P_{0}=$ maximum isometric tetanic force. For standard and long-term denervated grafts each variable was significantly different from the control EDL muscles in the old rats $(P<0.05)$. The asterisk indicates significant differences between the grafts or between the control EDL muscles in young and old rats.

dium pentobarbital $(50 \mathrm{mg} / \mathrm{kg})$ with supplemental doses as required. To isolate the muscle or graft, the skin covering the limb was cut around the ankle and then pulled up to the level of the upper thigh. The EDL muscle or graft was dissected free and the distal tendon was cut. The vascular and nerve connections to muscles and grafts were left intact. Suture (3-0) was tied to the distal tendon of the muscle or graft for subsequent attachment to the force transducer.

For stimulation of the muscle or graft by activation of the peroneal nerve, the sciatic nerve was exposed from knee to thigh. The common peroneal branch was dissected free from the rest of the nerve and severed in the region of the upper thigh. The length of peroneal nerve available for stimulation of the EDL muscle was 3 to 4 $\mathrm{cm}$. The branch of the nerve to the EDL muscle was left intact, and branches to all other muscles were cut.

With the rat on its side, the hindlimb was inserted into the bath through a hole in the side wall of the bath. The hole was sealed by stretching the skin of the hindlimb over a Plexiglas ring surrounding the hole and tying the skin in position. The knee and the ankle were fixed rigidly with metal screws. The bath contained approximately $200 \mathrm{ml}$ of mineral oil maintained at $35 \pm 1^{\circ} \mathrm{C}$. The peroneal nerve and the limb were maintained in the same mineral oil bath.

Isometric contractions of the EDL muscle or graft were obtained by supramaximal pulses of $0.2-\mathrm{ms}$ duration administered to either the peroneal nerve or through fine wire electrodes inserted into the proximal and distal tendons of the muscle. Force was measured by a Cambridge (305) ergometer. The ergometer was fastened to a rack and pinion device that permitted adjustment of muscle length. Muscle length was adjusted to give maximum twitch force of the whole muscle. The optimal length for force development $\left(L_{0}\right)$ was held con- stant for all subsequent measurements. Force development during isometric contractions was analyzed by a computer and plotted on an X-Y digital plotter. Forces between 50 and $5000 \mathrm{mN}$ could be recorded reliably. Peak twitch force $\left(P_{t}\right)$, time-to-peak twitch force (TPT), the frequency-force relationship, and the maximum isometric tetanic force $\left(P_{0}\right)$ were recorded for the whole muscle (8). Comparable data on contractile properties were obtained in vitro on six young (4.5 month) male F455 rats as a control for the in situ measurements. Significant differences among the groups of muscles were determined by a one-way analysis of variance. When the $F$ range was significant, group means were compared by $t$ tests, with significance accepted a priori at $P<0.05$. Data are presented as means \pm one $\mathrm{SE}$.

Following the measurement of contractile properties, each muscle was weighed, frozen in a mixture of dry ice and isopentane, and serially sectioned in a longitudinal plane. The sections were then stained for nerve fibers and motor end plates according to the combined silveracetylcholinesterase method of Goshgarian (11). For histological examination, regenerating muscles were fixed in Bouin's solution, embedded in paraffin, sectioned at $7 \mu \mathrm{m}$, and then stained with Ehrlich's hematoxylin and eosin.

\section{RESULTS}

\section{Histological Changes in Grafted Long-Term Denervated Muscle}

At the time of grafting the long-term denervated muscles were very thin - approximately the same diameter as the tendons. Two months after grafting the muscles remained thin (Table 1). The tissue connections between the grafts and tendon stumps were less robust 


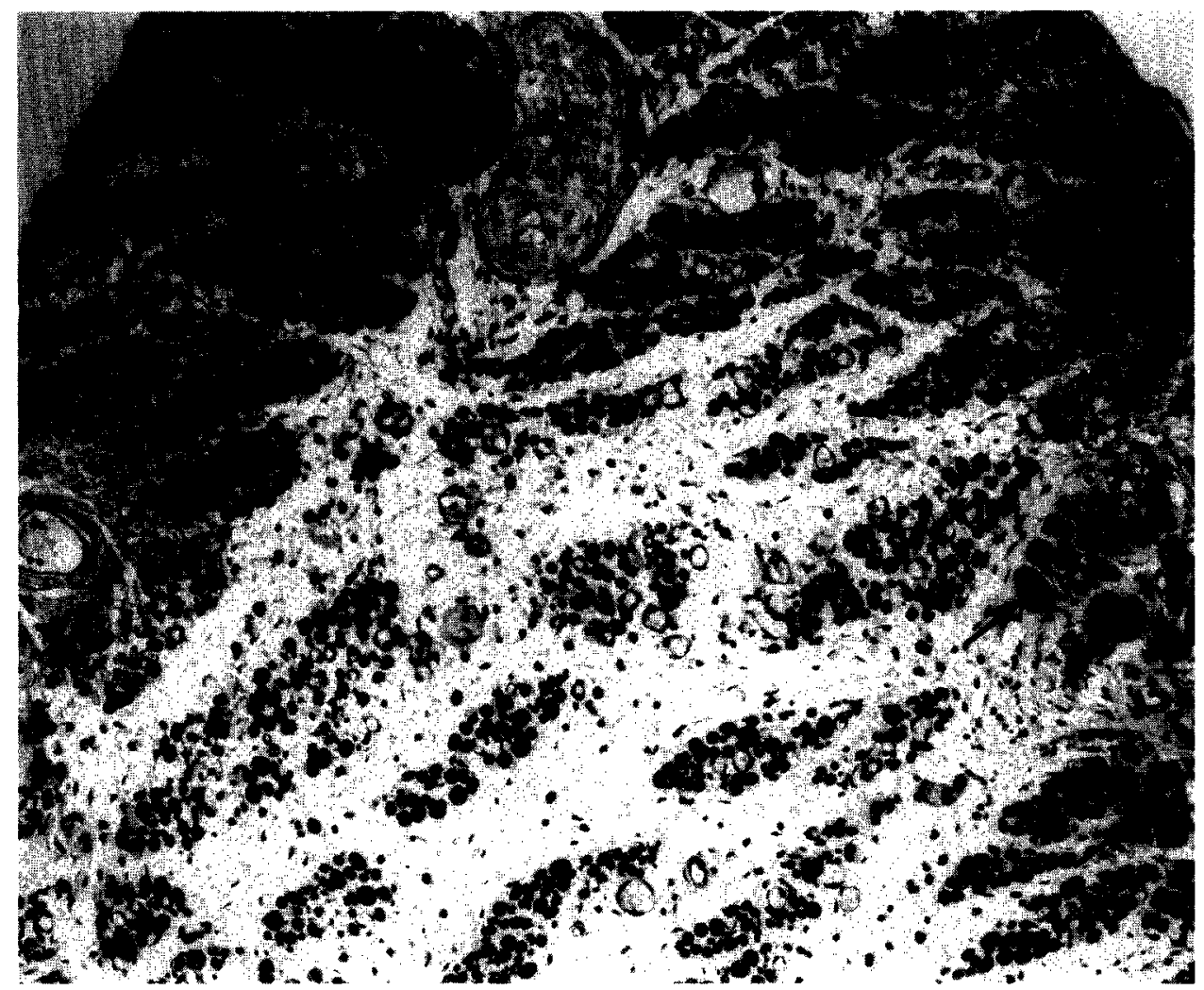

FIG. 2. Cross section through a 15-day graft of 22 -month denervated muscle. Islands of surviving muscle fibers (arrows) are seen among the regenerating ones. Hematoxylin and eosin. $90 \times$.

than those seen in standard grafts in young rats, but poor tendon regeneration is commonly seen in old rats (Carlson and Faulkner, unpublished observations). Histological examination of early muscle grafts revealed a complex pattern of highly atrophic surviving muscle fibers intermingled with regenerating muscle fibers (Fig. 2). The latter could be identified because of their cytoplasmic basophilia and, in longitudinal section, the characteristic chains of central nuclei. In older grafts it was not possible to distinguish regenerating from surviving muscle fibers with any degree of accuracy. Most grafts of long-term denervated muscle contained a proportion of loose interfascicular connective tissue higher than that of grafts of normal muscle (Fig. 2).

\section{The Return of Neuromuscular Function in Grafts of Long-Term Denervated Muscles and Their Controls}

Stimulation of the branch of the peroneal nerve that supplies the EDL muscle elicited a measurable contractile response from each of the long-term denervated and standard grafts. Compared to the maximum force developed by direct muscle stimulation, the maximum forces developed by nerve stimulation of control EDL muscles in young and old rats were 96 and $90 \%$, respectively. For the standard grafts in old rats nerve stimulation pro- duced a maximum force that was $81 \%$ of the value achieved with direct stimulation of the graft. Although the long-term denervated grafts contracted with nerve stimulation, we did not attempt to measure the maximum force developed. In each of the muscles on which contractile properties were measured, plus three other muscles that were not electrically stimulated, microscopic examination revealed the presence of acetylcholinesterase-positive motor end plates with silver-stained nerve fibers leading to them. Similarly, each of the six control grafts of normal EDL muscle contracted upon stimulation of the motor nerve, and each of these contained innervated motor end plates, as determined by histological criteria (Fig. 3).

\section{Contractile Properties of Grafts of Long-Term Denervated Muscles}

The TPTs of both types of grafts were not significantly different from the values of $19 \pm 1 \mathrm{~ms}$ obtained for control EDL muscles of 26 -month-old rats. Compared to the $P_{0}$ of $2414 \mathrm{mN}$ developed by control EDL muscles from old rats (Table 1 ), the values were $19 \%$ for standard grafts in old rats and $7 \%$ for long-term denervated grafts (Table 2). Compared to the standard grafts, the mass of the long-term denervated grafts was $66 \%$ and the maxi- 


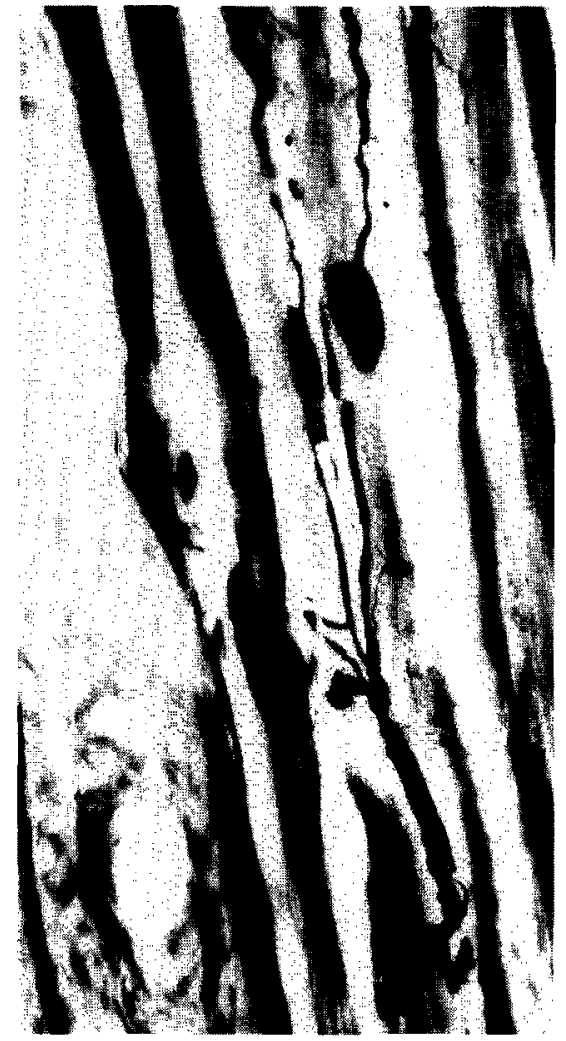

FIG. 3. Silver-acetylcholinesterase preparation of a frozen section showing a bundle of nerve fibers and motor end plates in a graft of long-term denervated muscle. Because of the section thickness (40 $\mu \mathrm{m})$ the nerve fibers are in focus, but the motor end plates and some of the nerve terminals are not. $154 \times$.

mum force was $40 \%$. The $P_{\mathrm{t}} / P_{0}$ ratio for both types of grafts was approximately 0.35 . This was significantly higher than the value of 0.26 obtained for control EDL muscle. The maximum specific forces $\left(\mathrm{N} / \mathrm{cm}^{2}\right)$ of the standard and long-term denervated grafts were signifcantly lower than the value of $21 \mathrm{~N} / \mathrm{cm}^{2}$ obtained for control EDL muscles from old rats (Table 2).

\section{DISCUSSION}

Our experiment showed that after 22 months of denervation, rat muscles consistently became functionally reinnervated after they had been grafted into the normally innervated contralateral limb. The data permit us to conclude that after grafting, long-term denervated (22 months) muscle fibers in the rat are capable of becoming functionally reinnervated. The methods of analysis by whole muscle contractile properties and by silver-AChEstained serial sections do not allow us to make conclusions about individual muscle fibers and their patterns of reinnervation. Certainly, not all of the muscle fibers in the grafts were reinnervated.
At least three factors may account for the incomplete reinnervation of these grafts. (i) Reinnervation is incomplete even in muscle grafts in young rats $(6,20)$. Although the number of muscle fibers in a freely grafted EDL muscle returns to normal levels (5), commonly only about half of the regenerated muscle fibers appear to be innervated. (ii) Reinnervation of muscle fibers in old rats may not be as complete as it is in young rats. Although clinical opinions vary regarding the efficacy of reinnervation in older individuals $(9,14)$, little information exists from studies on laboratory animals. (iii) Some muscle fibers in long-term denervated grafts may be refractory to regenerating motor nerve terminals. This hypothesis would be difficult to test on standard in vivo models. However, in cultures of individual muscle fibers taken from the flexor digitorum brevis muscles of the same rats as those used in the experiment reported here, a high percentage of the denervated muscle fibers became reinnervated by neurites from explants of fetal spinal cord (15).

Regeneration of skeletal muscle after long periods of denervation has also been shown by Carraro et al. (7) and Mussini et al. (16). Those investigators described regeneration in muscle fibers of the rat hemidiaphragm that had been denervated for up to 16 months. With the grafts of long-term denervated muscle, a mixture of both surviving and regenerating muscle fibers was observed. In fact, after short periods of predenervation (14 days), almost nine times as many muscle fibers survived free grafting as is the case after grafting normal muscle (5). Whether the increased survival of muscle fibers is due to their smaller size and a decrease in the diffusion distance for oxygen and nutrients or whether some intrinsic change in the denervated muscle fibers favors their sur-

\section{TABLE 2}

Comparisons of the Values for Muscle Mass, Maximum Tetanic Force $\left(P_{0}\right)$, and Maximum Specific Force $\left(P_{0}, \mathrm{~N} / \mathrm{cm}^{2}\right)$ for Standard Grafts in Young Rats, Standard Grafts in Aged Rats, and Long-Term Denervated Grafts in Aged Rats Expressed as a Percentage of the Value for Control EDL Muscles

\begin{tabular}{lccc}
\hline & Muscle mass & $P_{0}(\mathrm{~N})$ & $P_{0}\left(\mathrm{~N} / \mathrm{cm}^{2}\right)$ \\
\hline $\begin{array}{c}\text { Adult rats } \\
\text { Standard grafts/ } \\
\text { control muscle }\end{array}$ & 59 & 57 & 67 \\
$\begin{array}{c}\text { Old rats } \\
\text { Standard grafts/ } \\
\quad \text { control muscle }\end{array}$ & 47 & 19 & 35 \\
$\begin{array}{c}\text { Old rats } \\
\text { Long-term denervated } \\
\text { grafts/control } \\
\text { muscle }\end{array}$ & 31 & 7 & \\
$\begin{array}{c}\text { Control EDL muscles } \\
\text { Old rats/adult rats }\end{array}$ & 94 & 81 & 87 \\
\hline
\end{tabular}


vival intact remains to be determined. The histological findings on early stages of development of long-term denervated grafts in this study point toward the survival of a high percentage of the original denervated muscle fibers in these grafts.

The observation that in old rats the TPTs of 60-day standard and long-term denervated grafts were not significantly different from those of the control EDL muscles is consistent with previous results on 60-day standard grafts in young rats (10). The TPT of $19 \mathrm{~ms}$ was slightly prolonged compared to the value of $12 \mathrm{~ms} r e-$ ported by us for young Sprague-Dawley rats (10). The difference may be due to the temperature of $35^{\circ} \mathrm{C}$ in the present study compared to $37^{\circ} \mathrm{C}$ in the previous investigation or to an inherent difference in the TPTs of muscles from the different strains of rats. The difference in age is not likely a factor since the TPTs of EDL muscles of young and old Wistar rats (12) and of young and old C57BL/6 mice (4) were not significantly different.

The $P_{0}$ developed by the control EDL muscles of young rats was slightly greater than previous values for this muscle $(8,10,12)$. The lower values for the $P_{0}$ of muscles from old compared to young animals agrees with other data on both rats (12) and mice (4). The values for specific $P_{0}$ 's of $26 \mathrm{~N} / \mathrm{cm}^{2}$ and $21 \mathrm{~N} / \mathrm{cm}^{2}$ are within the range normally expected for skeletal muscles from young and old animals, respectively $(4,8,10,12)$. The difference of $18 \%$ between the specific $P_{0}$ of muscles of old compared to young rats observed in the present study supports the conclusion of Brooks and Faulkner (4) that in old animals a decrease occurs in the force-generating capacity per unit area of muscle. The mechanism responsible for the deficit in the generation of force with aging is not known.

As observed in previous studies of autografts $(8,10$, $17)$, both the standard grafts and the long-term denervated grafts in the old rats showed significant reductions in specific $P_{0}$ compared to the normal control value. An analysis of the architecture and composition of soleus grafts in rats has indicated significant increases in connective tissue and water content (17). The relative proportion of these noncontractile components was greater in standard grafts in old rats than in standard grafts in young rats and greater in the long-term denervated grafts than in standard grafts in old rats. The long-term denervated muscle regenerates a smaller mass of viable skeletal muscle fibers than the standard graft denervated at the time of grafting, but in addition the longterm denervated graft has a $40 \%$ increase in noncontractile tissue.

The $P_{0}$ 's developed by autografts in young and old rats in this study were significantly greater than those developed by the autografts in the study of Gutmann and Carlson (12). During the past 10 years, significant improvements have occurred in the techniques for the free grafting of whole skeletal muscles. One major difference in the technique of grafting was the implantation of the nerve in our grafts, whereas in the Gutmann and Carlson study nerve implantation into the grafts was not done and reinnervation occurred spontaneously (12). Although a direct comparison has not been made, implantation of the nerve appears to improve significantly the reinnervation of grafts (19). Whether or not improved reinnervation of the grafts can explain the four- to fivefold greater development of force by autografts placed in old and young rats, respectively, cannot be resolved by this investigation. The improvement in the results of the grafting procedure in the present investigation have made more apparent the significant difference between the recovery of structure and function in autografts made in young and old rats. A number of studies have reported a $P_{0}$ for autografts in young rats to be approximately $50 \%$ of the $P_{0}$ developed by control EDL muscles $(8,10)$. The $P_{0}$ developed by autografts in old rats in this study was $19 \%$ of the control value or $36 \%$ of the value for autografts in young rats. Apparently either old skeletal muscle fibers regenerate less well or fibers regenerate less well in old animals.

\section{ACKNOWLEDGMENTS}

This work was supported by NIH Grants DE 07687 and AG 06157.

\section{REFERENCES}

1. ANDERL, H. 1977. Cross-face nerve grafting-up to 12 months of seventh nerve disruption. Pages 241-277 in L. R. RUBIN, Ed. Reanimation of the Paralyzed Face. Mosby, St. Louis.

2. ANDERL, H. 1978. Nerve repair and crossover grafting in facial nerve palsy. Pages 259-264 in S. FREDRICKS AND G. S. BRODY, Eds., Symposium on the Neurologic Aspects of Plastic Surgery, Vol. 17. Mosby, St. Louis.

3. Bateman, J. E. 1962. Trauma to Nerves in Limbs. Saunders, Philadelphia.

4. BRooks, S. V., AND J. A. FAULKNER. 1988. Contractile properties of skeletal muscles from young, adult, and aged mice. J. Physiol. (London), in press.

5. CARLSON, B. M. 1976. A quantitative study of muscle fiber survival and regeneration in normal, predenervated, and Marcainetreated free muscle grafts in the rat. Exp. Neurol. 52: 421-432.

6. CARLSON, B. M., K. R. WAGNER, AND S. R. MAX. 1979. Reinnervation of rat extensor digitorum longus muscles after free grafting. Muscle Nerve 2: 304-307.

7. Carraro, U., D. Morale, I. Mussini, S. LuCke, M. Cantini, R. Betta, C. Catani, L. Libera, D. BetTo, and D. Noventa. 1985. Chronic denervation of rat hemidiaphragm: Maintenance of fiber heterogeneity with associated increasing uniformity of myosin isoforms. J. Cell Biol. 100: 161-174.

8. CôTÉ, C., AND J. A. FAULKNER. 1984. Motor unit function in skeletal muscle autografts of rats. Exp. Neurol. 84: 292-305.

9. Elkington, J. ST. C. 1944. Prognosis of peripheral nerve injuries. Proc. $R$. Soc. Med. 37: 547-551. 
10. FaulKNER, J. A., AND B. M. CARLSON. 1985. Contractile properties of standard and nerve-intact muscle grafts in the rat. Muscle Nerve 8: 413-418.

11. Goshgarian, H. G. 1977. A rapid silver impregnation for central and peripheral nerve fibers in paraffin and frozen sections. Exp. Neurol. 57: 296-301.

12. GutmanN, E., AND B. M. Carlson. 1976. Regeneration and transplantation of muscles in old rats and between young and old rats. Life Sci. 18: 109-114.

13. GUTMANN, E., AND J. Z. YounG. 1944. The re-innervation of muscle after various periods of atrophy. J. Anat. 78: 15-43.

14. HAMLIN, E., AND A. L. WATKINS. 1947. Regeneration in the ulnar, median and radial nerves. Surg. Clin. North Am. 27: 10521061.

15. JAY, J. 1987. Effects of Denervation and Neuronal Contact on the Distribution of Cell Surface Molecules on Single Rat Skeletal Mus- cle Fibers in Vitro. Ph.D. Thesis, University of Michigan, Ann Arbor.

16. Mussini, I., G. Favaro, and U. Carraro. 1986. Repetitive events of aneural fiber regeneration during long-term denervation of regenerated rat muscles. Pages 29-34 in M. FREY AND G. Freilinger, Eds., Proc. of 2nd Vienna Muscle Symposium. Facultas, Universitätsverlag, Vienna.

17. Segal, S. S., T. P. White, AND J. A. FAulkner. 1986. Architecture, composition, and contractile properties of rat soleus grafts. Am. J. Physiol 250: (Cell Physiol. 19) C474-C479.

18. Sunderland, S. 1978. Nerves and Nerve Injuries, 2nd ed. Churchill-Livingstone, Edinburgh.

19. White, T. P., J. F. Villanacci, P. G. Moraleas, S. S. Segal, AND D. A. Essig. 1984. Exercise-induced adaptations of rat soleus muscle grafts. J. Appl. Physiol. 56: 1325-1334.

20. WOMBLE, M. D. 1986. The clustering of acetylcholine receptors and formation of neuromuscular junctions in regenerating mammalian muscle grafts. Am. J. Anat. 176: 191-205. 